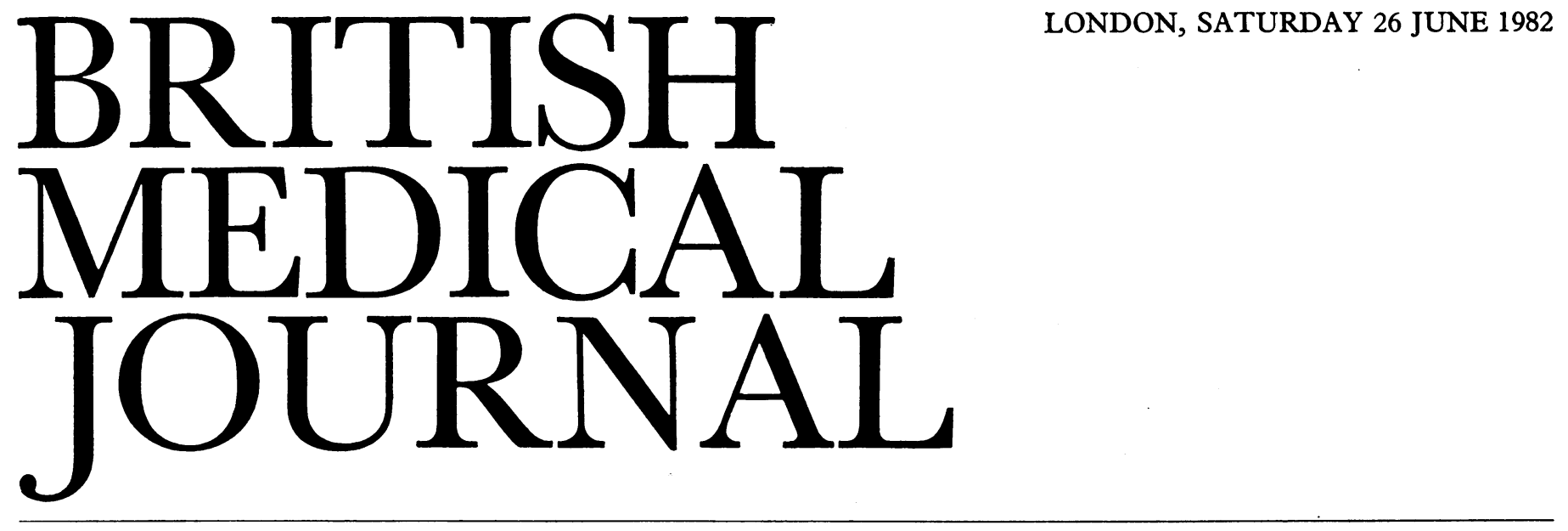

\title{
Less surgical and more medical infections in hospital?
}

Spectacular epidemics of hospital cross-infection have been investigated for 250 years, ${ }^{12}$ and were mainly due, in sequence, to the rickettsiae of typhus, Streptococcus pyogenes, Staphylococcus aureus, and various Gram-negative bacilli, notably Pseudomonas aeruginosa. During the past decade or so, however, epidemics have been largely replaced by less dramatic but insidiously pervasive endemic infections with a wide variety of opportunistic bacteria, fungi, viruses, and even some protozoa. The importance of the current problem is shown by the recent launch of the fournal of Hospital Infection, whose bright green cover emulates one of our leading hospital pathogens. Its second volume ended with a supplement giving the results of a survey of hospital infections in England and Wales. ${ }^{3}$

Previous national investigations of infections in over 3000 surgical ${ }^{4}$ and almost 7000 medical $^{5}$ patients had been carried out 25 and 20 years ago respectively. These had charted the incidence of hospital-acquired sepsis in a dozen hospitals over several months. Infections present when patients were admitted were excluded. In contrast, the new survey saved time and expense by recording on a single occasion the prevalence of infections-both those acquired in the community and those contracted in hospital-in over 18000 patients admitted to 43 hospitals (representing about one-seventh of the national daily inpatient population). Prevalence rates tend to be higher than incidence rates because infected patients generally stay in hospital longer than others. Nevertheless, interesting comparisons can be made over an interval of a quarter of a century-and yet, surprisingly, the authors of the latest report have not attempted to do so.

At first glance the depressing view is that infection is more rife in our hospitals today than it was 20 or more years ago. Over $19 \%$ of all patients were found to be infected in the recent survey compared with a combined total of only $7 \%$ in the two earlier studies. Apart from the higher weighting of prevalence data, however, infections have now been included when they were either present or incubating on admission, and these together accounted for just over half of all the cases. In other words, $9 \%$ of all the patients acquired infection in hospital. The highest rates were in special-care baby units $(16.8 \%)$, genitourinary surgery $(16 \cdot 1 \%)$, and, unexpectedly, orthopaedic surgery $(13.1 \%)$, with, at the other end of the scale, obstetrics $(4 \cdot 4 \%)$, paediatrics $(4 \cdot 1 \%)$, and ophthalmo$\log (2.1 \%)$.

The 1960 report on surgical wound infection had underestimated the problem by excluding any operations on the lower urinary tract, on the rectum or anus, and on accidental wounds; and it had also omitted any postoperative infections of the chest or other site away from the incision, as well as preexisting sepsis (other than appendix abscess or peritonitis). Thus the overall incidence of postoperative infection at that time was undoubtedly considerably higher than the $10 \%$ recorded for wounds alone, and the rate has now fallen to less than half this level. As might be expected, infection remains about three times more likely in contaminated operations $(12 \cdot 8 \%)$ than in "clean" surgical fields.

There is a hint in the latest report that the use of unspecified prophylactic antibiotics contributed appreciably to the reduction in the wound infection rate. But unfortunately the authors seem to have missed a splendid opportunity to correlate the use of different types of antimicrobial prophylaxis in a wide range of surgical procedures with the observed rates of wound infection-or indecd other forms of postoperative sepsis. When wound infection did occur Staph aureus wasperhaps surprisingly - the predominant pathogen, just as it had been 25 years ago at the height of the staphylococcal era. Nevertheless, the notoriously virulent "hospital staphylococcus" of $52 / 52 \mathrm{~A} / 80$ and related phage types, which caused pandemics as well as most endemic infections at that time, has been replaced by miscellaneous members of phage group III. The next most prevalent in both surgical surveys was Escherichia coli and then Proteus species. Streptococci figure next in the 1981 report, followed by relatively low rates of pseudomonas, klebsiella, and anaerobic infections. Unfortunately, culture results were available for only about half of the wounds judged to be infected on clinical grounds.

Away from the surgical wards, $8.2 \%$ of medical and acute geriatric patients were found to have acquired infection during their stay. After allowing for the different basis of the data, this prevalence rate still appears to compare unfavourably with the $5 \%$ incidence reported in $1965 . .^{3}$ The urinary tract is now the most frequent site of infections acquired in all adult medical and specialised surgical wards. Only in general surgery do wound infections predominate, followed by equally prevalent lower respiratory and urinary tract infections. Elsewhere in the hospital, lower respiratory infection is usually second in order of prevalence to urinary infection and at about half the latter's rate. This is a reversal of the picture 20 years ago, when the old "hospital staphylococcus" was implicated in at least half the pneumonias.

Skin infections rank third among all hospital-acquired sepsis 
considered together and second in the community-acquired infections. As with wounds, Staph aureus greatly outnumbers other pathogens at this site, but it is then followed by streptococci ahead of Gram-negative bacilli. The danger of bacterial dispersal from skin lesions as an often inapparent source of cross-infection requires constant vigilance. ${ }^{6}$

The principal site of infections already present on admission to hospital is the lower respiratory tract. When all types of community-acquired infections are considered together Staph aureus and $E$ coli each account for one-fifth of cases, followed among the bacteria, rather startlingly, by Mycobacterium tuberculosis $(8.4 \%)$, which is rivalled only by viruses $(9.6 \%)$. Candida species and miscellaneous fungi account for only $4 \cdot 1 \%$ and $0.3 \%$ respectively; but once in hospital the danger to immunocompromised patients posed by infection with these organisms-as with herpesviruses-is much greater than implied by their general prevalence rates.

The 1981 report mentions in a mere 11 words that it "reflects something of the added cost infection imposes on a hospital." In 1960 the cost of surgical sepsis alone in England and Wales was estimated at 1000000 days of excess hospital stay a year, with 5000 patients remaining in hospital for a month or more longer than expected and 500 or so dying from sepsis.

Things now seem better in relation to surgical sepsis, but most other forms of hospital-acquired infection appear to be at least as prevalent as they were 20 years ago. This is perhaps not surprising in our older and more debilitated hospital population. Many of these infections can and should be prevented, however, by rational measures based on adequately controlled trials. The recent survey points the way, but future investigations will require a more detailed and critical evaluation of the factors likely to be responsible and the effects of control procedures.

SYDNEY SELWYN

Professor of Medical Microbiology,

Westminster Medical School,

London SW IP 2AR

1 Selwyn S. Sir John Pringle: hospital reformer, moral philosopher and pioneer of antiseptics. Med Hist 1966;10:266-74.

2 Selwyn S. Sir James Simpson and hospital cross-infection. Med Hist 1965; 9:241-8.

3 Meers PD, Ayliffe GAJ, Emmerson AM, et al. Report on the National Survey of Infection in Hospitals, 1980. Fournal of Hospital Infection $1981 ; 2$, suppl:1-51.

4 Public Health Laboratory Service. Incidence of surgical wound infection in England and Wales. Lancet 1960 ;ii:659-63.

5 Public Health Laboratory Service. Infections acquired in medical wards. f Hyg Camb 1965;63:457-77.

6 Selwyn S, Chalmers D. Dispersal of bacteria from skin lesions: a hospital hazard. Br f Dermatol 1965;77:349-56.

\section{Bronchoalveolar lavage}

For more than 20 years lavage of the bronchial tree through a rigid bronchoscope has been used in the management of severe asthma $^{1}$ and of alveolar proteinosis. ${ }^{2}$ Once Myrvik et $a l^{3}$ had shown that in rabbits lavage could yield alveolar macrophages the technique formed the basis of the new topic of pulmonary cell biology, now rapidly developing and advancing our understanding of a wide variety of pulmonary diseases. The methods used for sampling the cells of the pulmonary inflammatory and immune systems of man have progressed through lavage via the rigid bronchoscope, ${ }^{4}$ or via a large balloon-tipped catheter wedged into a primary or secondary branch of the bronchial tree, ${ }^{56}$ to the present-day bronchoalveolar lavage via the fibreoptic bronchoscope-which was developed in the early $1970 s^{7-9}$ with only minor modifications since then.

Briefly, the fibreoptic bronchoscope is wedged in a sub- $\frac{2}{D}$ segmental bronchus and small quantities $(20-60 \mathrm{ml})$ of warmed, $\stackrel{2}{c}$ sterile, physiological saline are instilled through the biopsy $\widehat{C}$ channel of the bronchoscope and immediately aspirated into a $\overline{\bar{J}}$ sterile trap. ${ }^{1011}$ This procedure is repeated until a total of $100-$ D $300 \mathrm{ml}$ of saline has been instilled. Usually between $40 \%$ and $\$$ $70 \%$ of the infused volume is recovered, but in patients with destructive lung disease and airflow obstruction the proportion is smaller; recovery correlates inversely with the severity of $\stackrel{\vec{\rho}}{+}$ the airflow obstruction. ${ }^{12}$

Complications of bronchoalveolar lavage are rare. Transient $\frac{\bar{O}}{\bar{N}}$ respiratory distress and syncope have been reported, ${ }^{11}$ but $\frac{\bar{T}}{\sigma}$ these are also seen in patients undergoing fibreoptic broncho- $\mathbb{Q}$ scopy without bronchoalveolar lavage. The most frequent com- $ळ$ plication is fever, but even this is seen in fewer than $3 \%$ of $\overrightarrow{0}$ patients and rapidly responds to antibiotic treatment.

What are the present applications of bronchoalveolar lavage ? $\vec{\sigma}$ Clinically, it helps stage and monitor the progress of certain $\frac{5}{3}$ interstitial lung diseases. Untreated fibrosing alveolitis is characterised by the recovery of raised numbers of neutrophils ${ }^{13} \stackrel{\infty}{\circ}$ and eosinophils at bronchoalveolar lavage. These changes re- of flect the altered cellular populations found in specimens obtained by open-lung biopsy. ${ }^{14} 15$ Thus serial bronchoalveolar $\vec{\overrightarrow{ }}$ lavage may be used to assess progress of the disease. Repeat open-lung biopsy, in contrast, is impracticable, transbronchial $O$ biopsy has a substantial sampling error, ${ }^{16}$ and the standard ${ }_{N}^{2}$ pulmonary function tests or chest radiographs are less than of adequate for monitoring progress ${ }^{17}$; so bronchoalveolar lavage opens a prospect of better control of treatment. Even if serial ${ }^{\Phi}$ bronchoalveolar lavages are not performed studies (as yet to be $\vec{\varphi}$ independently confirmed) suggest that a single estimate of ? differential cell counts may be a useful prognostic indicator. ${ }^{16} 18$ O With pulmonary sarcoidosis, too, bronchoalveolar lavage appears to be helpful in assessing the activity of the disease. Sarcoidosis is characterised by recovery of increased percent-

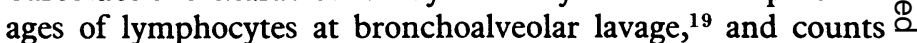
of the proportion of $\mathrm{T}$ lymphocytes, in conjunction with gal- $\overrightarrow{\overrightarrow{0}}$ lium-67 scanning, may predict which patients are likely to 3 deteriorate and so need treatment. ${ }^{20}$

Possibly of even greater importance than its direct clinical applications is the access that bronchoalveolar lavage provides to bronchoalveolar proteins and cells for research. For example, both pulmonary alpha $a_{1}$-antitrypsin ${ }^{21}$ and alveolar macro- $\frac{5}{3}$ phages, ${ }^{22}$ recovered by bronchoalveolar lavage, have been the subjects of recent studies of the development of emphysema in cigarette smokers. Similar applications of bronchoalveolar 0 lavage to pulmonary biochemistry and cell biology should ${ }_{N}$ provide new insights into this and other inflammatory and o immunological disorders of the lung.

Senior Registrar,

ANDREW P GREENING

Department of Respiratory Medicine,

Hammersmith Hospital,

London W 12 OHS

1 Thompson HT, Pryor WJ. Bronchial lavage in the treatment of obstructive $\mathbb{D}$ lung disease. Lancet 1964 ;ii:8-10.

2 Ramirez-RJ. Bronchopulmonary lavage. New techniques and observations. Diseases of the Chest 1966;50:581-8.

${ }^{3}$ Myrvik Q, Leake ES, Fariss B. Studies on pulmonary alveolar macro- $\mathbb{D}$ phages from the normal rabbit: a technique to procure them in a high $\mathbb{D}$ state of purity. $\mathcal{F}$ Immunol $1961 ; 86: 128-32$.

4 Keimowitz RI. Immunoglobulins in normal human tracheobronchial washings: a qualitative and quantitative study. F Lab Clin Med 1964; $63: 54-9$.

${ }^{5}$ Finley TN, Swenson EW, Curran WS, Huber GL, Ladman AJ. Bronchopulmonary lavage in normal subjects and patients with obstructive lung disease. Ann Intern Med 1967;66:651-8. 